\title{
The Mechanism of High Thermal Shock Resistance of Nanostructured Ceramic Coatings
}

\author{
Lihong Liang, ${ }^{*}$ Xiaona Li, and Yueguang Wei \\ LNM, Institute of Mechanics, Chinese Academy of Sciences, Beijing 100190, China
}

Hua Wei

Institute of Metal Research, Chinese Academy of Sciences, Shenyang 110016, China

The nanostructured and the conventional $\mathrm{ZrO}_{2}$ coating samples were thermal shocked at a series of temperatures. The elastic modulus and the hardness of two kinds of coatings were investigated by the nanoindentation tests. The results show that the corresponding mechanical properties of the conventional coatings increase monotonically with increasing temperature difference of the thermal shock. While the modulus and the hardness of the nanostructured coatings fluctuate slightly with increasing thermal shock temperature difference. Furthermore, the interface energy release model of the thermal shock strain energy was proposed for the nanostructured coatings. The theoretical prediction agrees with the experimental result.

\section{Introduction}

Ceramic coatings are widely used in biomedicine, aerospace, mechanical, chemical, and electrical engineering because of their excellent thermal insulation, wear resistance, and corrosion resistance. ${ }^{1}$ In some service conditions of ceramic coatings, thermal shock frequently occurs, for example, the blades of the gas turbine engines, with surface thermal barrier ceramic coatings, may experience abrupt temperature change above $1000 \mathrm{~K}$ during cooling from the hot gas stream, ${ }^{2}$ therefore, in-depth understanding of the mechanism of thermal shock resistance and the influence of the thermal shock on mechanical properties of the ceramic coating systems becomes significant. Some relevant studies on the thermal shock resistance of the thermal barrier coatings have been reported. Liang et al. investigated the thermal shock resistances of the nanostructured and the conventional zirconia coatings deposited by the atmospheric plasma spraying and found that the nanostructured coatings possess better thermal shock resistance than the conventional coatings, the endured thermal shock cycle number of the nanostructured coatings before failure increases about 2.5 times. $^{3}$ At the same time, the different microscopic fracture modes in the thermal shock, the transgranular (volume) fracture domination and the intergranular (interface) fracture domination for the conventional coatings and the nanostructured coatings, respectively, were found. ${ }^{3}$ Gerolamo

*lianglh@lnm.imech.ac.cn

(C) 2014 The American Ceramic Society et $a l^{4}$ also found the endured thermal shock cycle number of the nanostructured coatings increases about 2.5 times than the conventional coatings and attributed the higher thermal shock resistance to the special microstructure of the nanostructured coatings. Jamali et al. ${ }^{5}$ found the endured thermal shock cycle number of the nanostructured coatings increases about 1.5 times and attributed the higher thermal shock resistance to the lower elastic modulus and thus lower thermal stress. Song et $a l^{6}$ found the enhanced thermal shock resistance of the ceramics with the surface nanostructurization and attributed it to the hydrophobic structure of the surface. The lower thermal conductivity and the better thermal protect effect of the nanostructured coatings have been found, ${ }^{7,8}$ which explain the better thermal shock resistance partly. However, the mechanical mechanism of the increased thermal shock resistance of the nanostructured coatings is not clear due to the different reports of the elastic modulus of the nanostructured coatings. For example, different from the Jamali's report, Keyvani et $a l^{9}{ }^{9}$ found the higher elastic modulus of the as-sprayed nanostructured coatings compared with the as-sprayed conventional coatings.

As the elastic modulus is closely related to the thermal stress and the elastic strain energy produced in the thermal shock, the elastic modulus and the hardness of the thermal barrier coatings have been studied in many works, ${ }^{9-13}$ the change of the elastic modulus with annealing temperature and time, ${ }^{12,13}$ and the change with the thermal cycling have been reported. ${ }^{11}$ The elastic modulus increases intuitively with heating tempera- 
ture and thermal cycling, as the sintering of ceramics occurs usually during heating. ${ }^{11-13}$ Moreover, Keyvani et al. ${ }^{9}$ found the increase of the elastic modulus of the nanostructured coatings with increasing heat treatment time is weaker than the conventional coatings, and thus, the modulus of the nanostructured coatings is lower than the conventional coatings after the heat treatment despite the higher modulus of the as-sprayed nanostructured coatings. However, the effect of thermal shock on the elastic modulus of the ceramic coatings is rarely reported. What is ever the intrinsic relation between the mechanical properties and the thermal shock resistance, and what is the mechanism underlying the higher thermal shock resistance of the nanostructured coatings? The answers to the questions provide not only the guide in the application of the nanostructured coatings, but also the promotion in the scientific understanding of nanoscale thermal and mechanical coupling behavior.

In this study, the effects of the thermal shock on the elastic modulus and the hardness of the nanostructured coatings were compared with those of the conventional coatings, and the corresponding interface energy release model was proposed, based on the competition of the elastic strain energy produced in the thermal shock and the inner interface fracture energy, to reveal the mechanism underlying the higher thermal shock resistance of the nanostructured coatings.

\section{Experimental}

The thermal barrier coatings samples used in this study consist of the YSZ (8 wt $\% \mathrm{Y}_{2} \mathrm{O}_{3}$ stabilized $\mathrm{ZrO}_{2}$ ) top coats prepared by the standard atmospheric plasma spraying method, ${ }^{8,14}$ the NiCrAlY $(25.42 \mathrm{wt} \% \mathrm{Cr}-5.1 \mathrm{wt}$ $\% \mathrm{Al}-0.48 \mathrm{wt} \% \mathrm{Y}$ ) bond coats prepared by the high velocity oxygen fuel method, and the Ni-based superalloy substrates. The detailed preparation process and parameters are referred to Ref. 14. The thicknesses of the top coats, the bond coats, and the substrates are approximately $0.15 \mathrm{~mm}, 0.05 \mathrm{~mm}$, and $2.8 \mathrm{~mm}$, respectively. The diameter of the samples is about $15 \mathrm{~mm}$. Two kinds of ceramic coatings were prepared using different raw powders, one kind of coatings possess the grains of about 40 $100 \mathrm{~nm}$ diameter prepared from the nanostructured YSZ powder, ${ }^{8}$ and the other kind is the conventional coatings with bulk splat grains of $200 \mu \mathrm{m}$ diameter and $2 \mu \mathrm{m}$ thickness prepared from the conventional YSZ powder. ${ }^{2,8}$ The porosity of the nanostructured coatings and the conventional coatings is $8 \%$ and $14 \%$, respectively. ${ }^{8}$ At least, 12 samples were prepared for each kind of coatings for the thermal shock test of different temperatures. The microstructure of the samples was observed by the FEI Sirion 400 NC scanning electron microscope (SEM). The average porosity of the coatings after the thermal shock was estimated by image analyzing based on the cross-section micrographs of the coatings, and the average value of at least three micrographs (with the smallest area of about $60 \mu \mathrm{m} \times 50 \mu \mathrm{m}$ ) for each sample was taken.

Two kinds of coating samples (three samples for each kind for each test) were heated with a rate of $20 \mathrm{~K} /$ min up to a preset temperature $T_{h}(473 \mathrm{~K}, 773 \mathrm{~K}$, and $1073 \mathrm{~K}$, respectively) in the KSW5-12 box resistance furnace and held at $T_{h}$ for $20 \mathrm{~min}$. After that, the heated samples were quickly placed into water at the ambient temperature (the room temperature $298 \mathrm{~K}$ ) for quenching and maintained for $10 \mathrm{~min}$. Therefore, the temperature difference $\Delta T$ of the thermal shock is $175 \mathrm{~K}$, $475 \mathrm{~K}$, and $775 \mathrm{~K}$, respectively.

The coating surface of the samples, including the assprayed samples and the samples after the thermal shock, was ground and polished, then the mechanical properties of the coatings were measured by the nanoindentation tests using the TriboIndenter Nanomechanical Test Instrument (HYSITRON) based on the classical Oliver and Pharr method. ${ }^{15}$ All samples were ground and polished to the same degree, and the tests were all conducted at a same depth (about $15 \mu \mathrm{m}$ ) from the outer surface of the samples. All indentations were carried out with a triangular pyramid Berkovich diamond indenter. The total number of measurement points for each sample is 20 , and the measurement points were distributed as possible as uniformly on the surface of the coatings. The indentation depth is $300 \mathrm{~nm}$, and the maximum load is $3 \mathrm{mN}$ in the test. The rate of the indentation is $600 \mu \mathrm{N} / \mathrm{s}$. The loading time is $10 \mathrm{~s}$. The typical distance between two neighboring sites is above $50 \mu \mathrm{m}$ aiming to avoid possible interference of measurements. During the indentation test, the indentation load and depth were measured by the load cell and the gap sensors. The elastic modulus and the hardness were calculated based on the load-depth curves, and the average value of 20 measured data were taken for each sample. Moreover, the Vickers hardness of the coatings was measured using the microhardness tester $\mathrm{MH}-6$ under the load $9.8 \mathrm{~N}$ to compare with the results of the nanoindentation test.

\section{Results and Discussions}

Figure 1 shows the change of the elastic modulus $E$ and the hardness $H$ of the nanostructured and the conventional coatings with the thermal shock temperatures based on the results of the nanoindentation tests. It can 


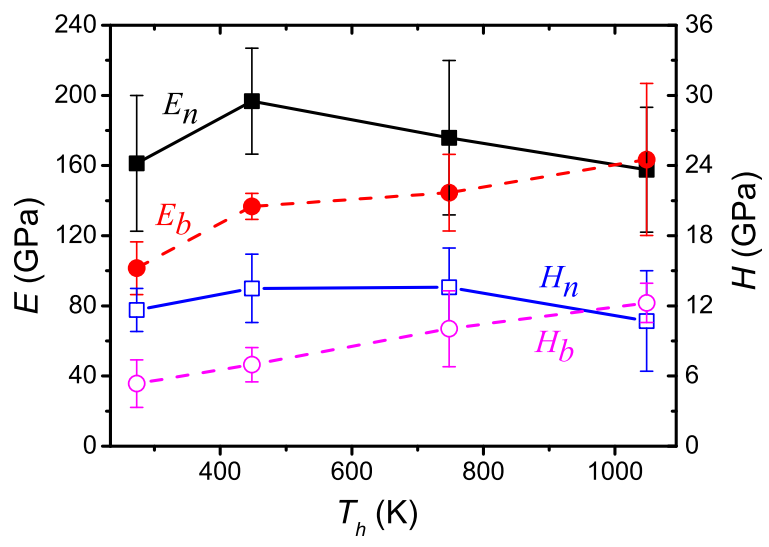

Fig. 1. The change of the elastic modulus $E$ and the hardness $H$ of the coatings with heating temperature of the thermal shock, the subscripts $n$ and $b$ represent the nanostructured and the conventional coatings, respectively.

be seen from that after the thermal shock in the heating temperature range from $473 \mathrm{~K}$ to $1073 \mathrm{~K}, E_{n}$ and $H_{n}$ of the nanostructured coatings fluctuate slightly with increasing temperature difference of the thermal shock, while $E_{b}$ and $H_{b}$ of the conventional coatings increase monotonously with increasing temperature difference. For the as-spayed coatings before the thermal shock, the average Young's modulus of the nanostructured coatings $E_{n}$ and the conventional coatings $E_{b}$ are in agreement with previous reports well ${ }^{7,16-18}$ as shown in Table I; the average hardness of the nanostructured coatings $H_{n}$ and the conventional coatings $H_{b}$ are also close to the data in the literatures ${ }^{15,18}$ showed in Table I. The elastic modu-

Table I. Comparisons of $E$ and $H$ of the as-sprayed Nanostructured and Conventional Coatings Between our Test Results and the Results from Other Groups

\begin{tabular}{lrrrrr}
\hline & $\boldsymbol{E}(\mathbf{G P a})$ & \multicolumn{3}{c}{$\boldsymbol{H}(\mathbf{G P a})$} \\
\hline Nano. (n) & $161.2^{*}$ & $155^{7}$ & $160^{16}$ & $11.6^{*}$ & 10.5 \\
Conven. (b) & $101.4^{*}$ & $96.4^{17}$ & $94^{18}$ & $5.4^{*}$ & 4.2 \\
\hline
\end{tabular}

* Our measured average value. lus and the hardness of the as-sprayed nanostructured coatings are both higher than those of the as-sprayed conventional coatings. The measurements of the microhardness show the same result and the same trend with the thermal shock temperature. Note that no matter which method, the nanoindentation ${ }^{13}$ or the Vickers and Knoop indentations, ${ }^{11}$ the change trend of the modulus and the hardness with the annealing time or the thermal cycle number is the same.

Although the elastic modulus of the nanostructured coatings is higher than that of the conventional ones for the as-sprayed coatings due to the smaller porosity, $E_{n}$ is lower than $E_{b}$ after the thermal shock of about $800 \mathrm{~K}$ as shown in Fig. 1, which may be resulted from the different microstructure changes with the thermal shock temperatures. For the conventional coatings, the increased thermal stress and the elastic strain energy, which is proportional to the elastic modulus, would be produced in the sequent thermal shock cycle as the elastic modulus increases after the forestalled thermal shock. But for the nanostructured coatings, the thermal shock stability of the elastic modulus would improve the thermal stress and the thermal strain energy in the sequent thermal shock cycle, which is similar to the previous report that the thermal stress and the elastic modulus of the nanostructured coatings are lower than those of the conventional coatings with the thermal oxidation time although the initial thermal stress and the elastic modulus of the nanostructured coatings are higher.'

As above mentioned, the elastic modulus difference of the ceramic coatings should be related with the microstructure difference. Figure 2 shows the representative cross-section micrographs of the as-sprayed nanostructured coating (Fig. 2a) and the conventional coating (Fig. 2b), it can be seen that the porosity of the former is lower than that of the latter, which explains the higher initial elastic modulus of the nanostructured coatings as shown in Fig. 1. For the as-sprayed nanostructured coatings and the conventional coatings, the porosity-dependent modulus can be expressed as

Table II. The Porosity of the Conventional and Nanostructured Coatings after the Thermal Shock based on the Cross-Section Micrographs (SEM) and the Image Analyzing

\begin{tabular}{lllll}
\hline $\boldsymbol{\Delta} \boldsymbol{T}(\mathbf{K})$ & $\mathbf{0}$ & $\mathbf{1 7 5}$ & $\mathbf{4 7 5}$ & $\mathbf{7 7 5}$ \\
\hline Conven. (b) & $0.140 \pm 0.003$ & $0.095 \pm 0.003$ & $0.086 \pm 0.001$ & $0.081 \pm 0.008$ \\
Nano. $(n)$ & $0.084 \pm 0.005$ & $0.068 \pm 0.011$ & $0.138 \pm 0.013^{*}$ & $0.050 \pm 0.018$ \\
\hline
\end{tabular}

${ }^{*}$ The value may not be representative as it is the result of the spare sample due to the previous failure of preparing SEM sample and collecting micrograph. 

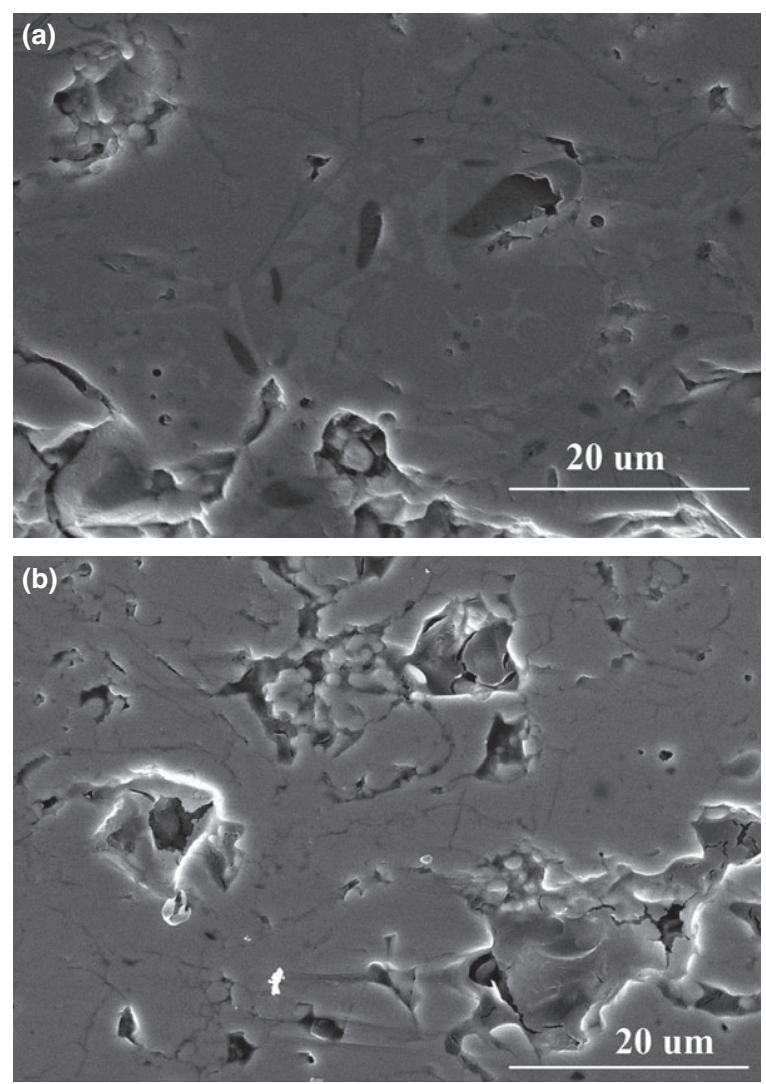

Fig. 2. The representative cross-section micrographs of the assprayed nanostructured (a) and the conventional (b) coatings obtained by the SEM showing the smaller porosity of the nanostructured coatings.

$$
\begin{aligned}
& E_{n}=E_{0} \exp \left(-a p_{n}\right), \\
& E_{b}=E_{0} \exp \left(-a p_{b}\right),
\end{aligned}
$$

respectively, based on the simple, classical expression of porous materials, ${ }^{15} E_{0}$ is the elastic modulus for the dense ceramics, $a$ is a constant related to the materials, and $p$ is the porosity. It can be seen from Fig. 1 that $E_{n}=161 \mathrm{GPa}$ and $E_{b}=101 \mathrm{GPa}$ for the as-sprayed coatings, combining with $p_{n}=0.08$ and $p_{b}=0.14,{ }^{8}$ by substituting these quantities into Eqs. (1.1) and (1.2), $a=7.7$ and $E_{0}=298 \mathrm{GPa}$ can be obtained, the result of the Young's modulus is in agreement with the calculated result of $\mathrm{ZrO}_{2}$ crystals without porosity. ${ }^{19}$

When the effect of the thermal shock is considered, which may change the microstructure and the porosity of the coatings and thus change the elastic modulus, substituting parameters $a$ and $E_{0}$ into Eq. (1.2) for the conventional coatings, the following equation can be obtained

$$
E_{b}(\Delta T)=298 \times \exp \left[-7.7 p_{b}(\Delta T)\right],
$$

where $\Delta T$ in the parentheses represents the temperature difference of the thermal shock. For the as-sprayed conventional coatings before the thermal shock, that is, when $\Delta T=0, \quad E_{b}(0)=101 \mathrm{GPa}$ and $p_{b}(0)=0.14$. When $\Delta T=775 \mathrm{~K}, E_{b}(775)=164 \mathrm{GPa}$ can be seen from Fig. 1, by substituting the value into Eq. (2), $p_{b}(775)=0.08$ can be obtained, agreeing with our experimental observation well as shown in Table II. Table II shows the porosity change of the coatings with the thermal shock temperature. It can be seen that the average porosity of the conventional coatings decreases monotonically with increasing temperature difference of the thermal shock, which is reasonable as the sintering occurs usually during heating before the quenching and the microstructure becomes denser, ${ }^{11,13,20}$ which explains the monotonous increase of the elastic modulus of the conventional coatings with the thermal shock temperature as shown in Fig. 1. Interestingly, the porosity of the conventional coatings after the thermal shock of about $800 \mathrm{~K}$ is the almost same as that of the as-sprayed nanostructured coatings as shown in Table II, which also explains the reason that they have close elastic modulus as shown in Fig. 1.

Figure 3 shows the change of the elastic modulus of the coatings with changing porosity, it can be seen that the relation between the measured elastic modulus and the porosity for the conventional coating agrees with the prediction in terms of Eq. (2) well. While for the nanostructured coatings, the disperseness of the experimental data, especially for the data after the higher temperature

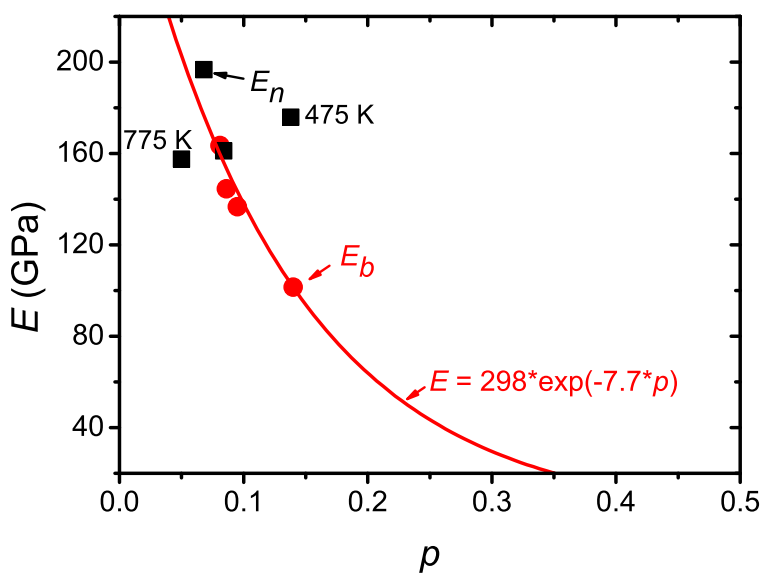

Fig. 3. The change of the average elastic modulus $E$ with the average porosity $p$. The curve is the prediction based on Eq. (2), the circles and the squares are measured results for the conventional and the nanostructured coatings, respectively. 
thermal shock ( $475 \mathrm{~K}$ and $775 \mathrm{~K}$ ), is larger, this is understandable as there are larger errors and uncertainty in the porosity of the nanostructured coatings after the thermal shock of $475 \mathrm{~K}$ as shown in Table II. At the same time, the porosity of the nanostructured coatings is smaller and the more microcracks along the particles' interfaces appear after the thermal shock of $475 \mathrm{~K}$ as shown in Fig. 4, the microcracks affect the elastic modulus greatly. Note that there is no change nearly in the elastic modulus of the nanostructured coatings after the thermal shock of about $800 \mathrm{~K}$, although the porosity of the coatings decreases, which may be attributed to the more inner interfaces of the nanostructured coatings and the microcracks induced by the thermal shock of the higher temperature. The microcracks can decrease the elastic modulus importantly, ${ }^{13,21,22}$ the detailed microcrack effect on the elastic modulus beside the porosity effect can be referred to Ref. 21,22. On the other hand, the microcracks correspond to the inner interface fracture induced by the produced elastic strain of the nanostructured coatings in the thermal shock process as the grain size is smaller and the total interface area is larger, and the inner interface fracture is favorable to release the thermal shock strain energy.

The microstructure of the nanostructured coatings is the nanometer-scale grains of average diameter $70 \mathrm{~nm},{ }^{8}$ while the bulk splat grains of the conventional coatings are about $200 \mu \mathrm{m}$ diameter and $2 \mu \mathrm{m}$ thickness, ${ }^{2,8}$ the total inner interface area of the nanostructured coatings increases about two orders than that of the conventional ones in the same total volume of the coatings; therefore, the elastic strain energy of the nanostructured coatings produced in the thermal shock is easy to be released by the inner interface fracture corresponding to the micro-

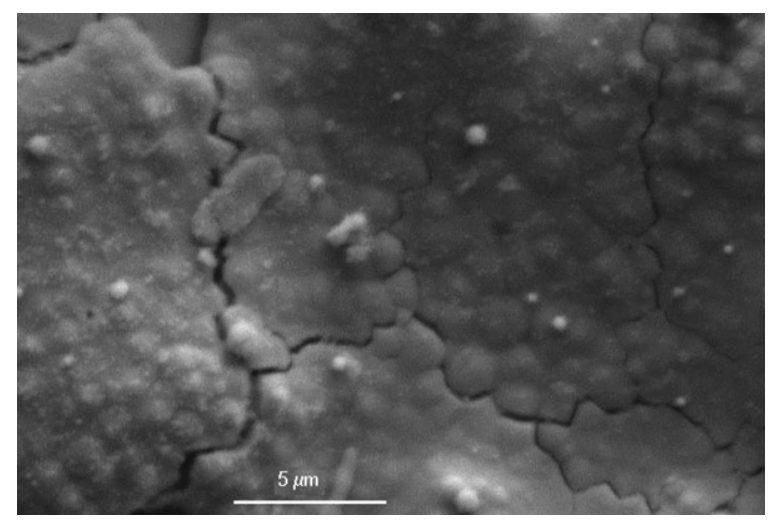

Fig. 4. The representative surface micrograph of the nanostructured coating after the thermal shock of $475 \mathrm{~K}$ obtained by the SEM showing the more microcracks along the interfaces of the particles. cracks. The total energy change $\Delta U$ of the ceramic coatings after the thermal shock can be expressed as

$$
\Delta U=U_{e} V-n \gamma_{i} A_{g} / 2,
$$

based on the competition between two kinds of energies, where $U_{e}$ is the elastic strain energy per unit volume produced in the thermal shock, $V$ is the total volume of the coatings, $\gamma_{i}$ is the released interface energy per unit area by the grain boundary fracture between two neighbor grains, $n$ is the number of grains in the coatings, and $A_{g}$ is the surface area of one grain. According to the thermodynamic laws, when $\Delta U<0$, the process occurs spontaneously, that is, the elastic energy produced in the thermal shock can be released by the enough inner interface fracture. Otherwise, the elastic energy is released by the grain volume fracture, which will lead to the more serious break of the coatings.

The elastic strain energy $U_{e}$ per unit volume of the ceramic coatings produced in the thermal shock can be expressed as

$$
U_{e}=\frac{1}{2} E(\alpha \Delta T)^{2},
$$

simply, where $\alpha=10 \times 10^{-6} / \mathrm{K}$ is the thermal expansion coefficient of the ceramic coatings. ${ }^{1}$ By substituting $E_{n}=161 \mathrm{GPa}$ and $\Delta T=775 \mathrm{~K}$ into Eq. (4), the strain energy of the nanostructured coatings $U_{e n}=4.84 \mathrm{MPa}$ can be obtained. Combining with the total volume of the coatings $V=2.65 \times 10^{-8} \mathrm{~m}^{3}$ calculated by the diameter of the samples and the thickness of the coatings, $U_{e n} V$ is the order of $10^{-1} \mathrm{~J}$ for the nanostructured coatings as shown in Table III. For the conventional coatings, substituting $E_{b}=101 \mathrm{GPa}$ and $\Delta T=775 \mathrm{~K}$ into Eq. (4), $U_{e b}=3.05 \mathrm{MPa}$, combining with the same total volume of the coatings $V, U_{e b} V$ is the order of $10^{-2} \mathrm{~J}$ as shown in Table III. Although the total elastic strain energy of the conventional coatings is smaller than that of the nanostructured coatings due to the smaller elastic modulus, the strain energy of one grain of the conventional coatings is much larger than that of the nanostructured coatings due to the much larger grain volume.

The interface fracture energy should be the energy difference between two surface energies after the fracture and the interface energy before the fracture strictly, ${ }^{14}$ considering the close value of the surface energy and the interface energy (several Joules per square meter), the interface fracture energy per unit area between two neighbor grains here can be approximately taken as the grain boundary energy simply, that is, the solid-solid interface energy. The solid-solid interface energy can be calculated by the related thermodynamic parameters of 
Table III. Comparison of the Strain Energy and the Interface Energy between the Nanostructured and the Conventional Coatings

\begin{tabular}{llll}
\hline & $\begin{array}{l}\text { Volume } \\
\text { strain } \\
\text { energy- } \boldsymbol{U}_{\boldsymbol{e}} \boldsymbol{V}(\mathrm{J})\end{array}$ & $\begin{array}{l}\text { Interface } \\
\text { release } \\
\text { energy- } \\
\boldsymbol{n} \gamma_{\boldsymbol{i}} \boldsymbol{A} / \mathbf{2}(\mathrm{J})\end{array}$ & $\begin{array}{l}\text { Total } \\
\text { energy } \\
\text { change- } \\
\boldsymbol{\Delta} \boldsymbol{U}(\mathbf{J})\end{array}$ \\
\hline Nano. $(n)$ & 0.13 & 5.29 & $-5.16<0$ \\
Conven. $(b)$ & 0.08 & 0.06 & $0.02>0$ \\
\hline
\end{tabular}

the corresponding crystals, ${ }^{23} \gamma_{\mathrm{i}}=4 h S_{v} H /\left(3 V_{m} R\right)$, where $h=0.324 \mathrm{~nm}$ is the average bond length of the $\mathrm{ZrO}_{2}$ crystals (calculated by the lattice volume ${ }^{19}$ ), $S_{v}=S_{m}-R$ is the vibrational part of the melting entropy with the melting entropy $S_{m}=H / T_{m}{ }^{24} T_{m}=2973 \mathrm{~K}$ is the melting point, ${ }^{2} H=87,500 \mathrm{~J} / \mathrm{mol}$ is the melting enthalpy, ${ }^{25} \quad R$ is the ideal gas constant, and $V_{m}=20.54 \mathrm{~cm}^{3} / \mathrm{mol}$ is the molar volume of the crystal (calculated by the mass and the density ${ }^{2}$ ). According to the above equation, $\gamma_{i}=4.67 \mathrm{~J} / \mathrm{m}^{2}$ can be calculated for the ceramic coatings. For the nanostructured coatings, the surface area $A_{g n}=1.54 \times 10^{-14} \mathrm{~m}^{2}$ for one grain, and the number of the grains in the coatings $n_{n}$ $=1.47 \times 10^{14}$, thus the total interface energy $n_{n} \gamma_{i} A_{g n} l$ 2 is about $5 \mathrm{~J}$ as shown in Table III, which is more than one order larger than the elastic strain energy of 0.13 , therefore, according to Eq. (3), $\Delta U<0$, that is, the elastic energy produced in the thermal shock can be released by the interface fracture. However, for the conventional coatings, the surface area $A_{g b}=6.41 \times$ $10^{-8} \mathrm{~m}^{2}$ for one grain, and the number of the grains $n_{b}=4.22 \times 10^{5}$, the total interface energy $n_{b} \gamma_{i} A_{g b} / 2$ is the order of $10^{-2} \mathrm{~J}$ as shown in Table III, which is smaller slightly than the elastic strain energy, according to Eq. (3), $\Delta U>0$, that is, the elastic energy produced in the thermal shock is not easy to be released by the interface fracture, but tends to be released by the volume fracture. In fact, the different microscopic fracture modes, the transgranular (volume) fracture domination, and the intergranular (interface) fracture domination for the conventional coatings and the nanostructured coatings, respectively, in the thermal shock have been found experimentally (fig. 7 in Ref. 3), which are in agreement with our analysis. And the interface energy releasing mechanism can explain quantitatively the experiment results well, which also explains why the nanostructured coatings have the better thermal shock resistance. $^{3}$

When the effective spherical grains with the diameter $D$ are considered, by substituting Eq. (4) into
Eq. (3), the following expression can be obtained

$$
\Delta U=\frac{V}{2}\left[E(\alpha \Delta T)^{2}-6 \gamma_{i} / D\right] .
$$

Equation (5) indicates that the total energy change of the coatings after the thermal shock increases with increasing thermal shock temperature and the size of the microstructure when the elastic modulus, the thermal expansion coefficient, the interface energy, and the volume of the coatings are constants as shown in Fig. 5. At the certain thermal shock temperature, the total energy change decreases with decreasing microstructure size; therefore, the nanostructured coatings are favorable to release the thermal shock strain energy by the inner interface cracking, and the critical grain size satisfying $\Delta U=0$ can be calculated. For example, when $\Delta T=775 \mathrm{~K}$, the critical effective diameter $D$ is about $2 \mu \mathrm{m}$ based on Eq. (5), that is, when size of the effective spherical gains is smaller than this critical size, the thermal shock strain energy of the coatings can be released by the enough inner interface fracture. When the thermal shock temperature increases, the critical size decreases. On the other hand, the elastic modulus of the conventional coatings increases with the thermal shock temperature; therefore, the total energy change increases in the next thermal shock, and thus, the thermal shock resistance is lower. Note that thermal mismatch between the ceramic coatings and the alloy substrates is not considered here, only the properties of the ceramic coatings is discussed. The interface thermal mismatch between the nanostructured coatings and the substrates will be

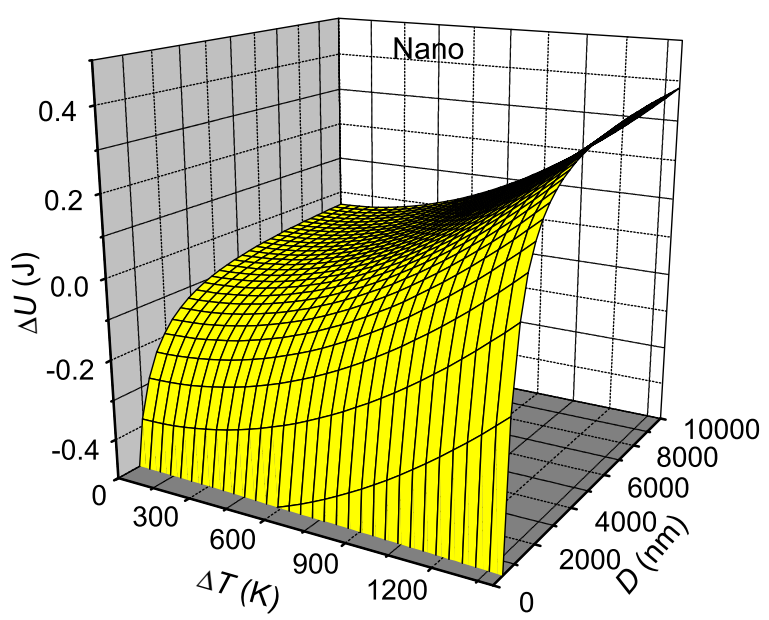

Fig. 5. The total energy change $\Delta U$ of the coatings as the function of the thermal shock temperature difference $\Delta T$ and the effective diameter $D$ of the coating microstructure in terms of Eq. (5). In Eq. (5), the elastic modulus is taken as the value of the assprayed nanostructured coatings. 
studied in further work. The thermal expansion coefficient of the nanostructured coatings was assumed to be same as that of the conventional ones, even if the thermal expansion coefficient is different, as long as the difference is not obvious, there is no obvious change in the thermal strain energy.

\section{Conclusion}

In summary, the change of the elastic modulus and the hardness of the air plasma sprayed ceramic coatings after the thermal shock of different temperatures was investigated by the nanoindentation and the microhardness tests. The results show that the Young's modulus and the hardness of the nanostructured coatings fluctuate slightly as the temperature difference of the thermal shock increases, while those of the conventional coatings monotonously increase with increasing thermal shock temperature difference. The stability of thermal shock of the elastic modulus of the nanostructured coatings is resulted from the more inner interfaces and enough interface energy releasing, and the enhancement of the elastic modulus of the conventional coatings is originated from the decrease of the porosity. The different energy releasing mechanisms explain the different microscopic fracture modes for the nanostructured coatings and the conventional ones. Therefore, in the temperature range we studied, the mechanical properties of the nanostructured coatings are less sensitive to the change of temperature, which makes the nanostructured coatings present the better resistance against the thermal shock.

\section{Acknowledgments}

This work was supported by the National Basic Research Programs of China (No. 2012CB937500), the NSFC grants of China (Nos. 11023001, 11372318,
11432014, and 91216108), the CAS/SAFEA International Partnership Program for Creative Research Teams and the opening fund of LNM.

\section{References}

1. A. G. Evans, D. R. Mumm, J. W. Hutchinson, G. H. Meier, and F. S. Pettit, Prog. Mater Sci., 46 505-553 (2001).

2. N. P. Padture, M. Gell, and E. H. Jordan, Science, 296 280-284 (2002).

3. B. Liang and C. Ding, Surf. Coat. Technol., 197 185-192 (2005).

4. G. Di Girolamo, F. Marra, C. Blasi, E. Serra, and T. Valente, Ceram. Int., 37 2711-2717 (2011).

5. H. Jamalin, R. Mozafarinia, R. S. Razavi, and R. Ahmadi-Pidani, Ceram. Int., 38 6705-6712 (2012).

6. F. Song, S. Meng, X. Xu, and Y. Shao, Phys. Rev. Lett., 104125502 (2010).

7. G. Soyez, et al., Appl. Phys. Lett., 77 1155-1157 (2000).

8. L. H. Liang, H. Wei, X. C. Chang, W. Xu, X. N. Li, and Y. G. Wei, Int. J. Heat Mass Transf., 65 219-224 (2013).

9. A. Keyvani, M. Saremi, M. Heydarzadeh Sohi, and Z. Valefi, J. Alloys Compd, 541 488-494 (2012).

10. R. S. Lima, A. Kucuk, and C. C. Berndt, Surf. Coat. Technol., 135 166-172 (2001).

11. J. P. Singh, B. G. Nair, D. P. Renusch, M. P. Sutaria, and M. H. Grimsditch, J. Am. Ceram. Soc., 84 [10] 2385-2393 (2001).

12. S. R. Choi, D. M. Zhu, and R. A. Miller, J. Am. Ceram. Soc., 88 [10] 2859-2867 (2005).

13. N. Zotov, M. Bartsch, and G. Eggeler, Surf. Coat. Technol., 203 2064-2072 (2009).

14. L. H. Liang, H. Wei, X. N. Li, and Y. G. Wei, Surf. Coat. Technol., 236 525-530 (2013).

15. B. K. Jang and H. Matsubara, Mater. Lett., 59 3462-3466 (2005) and reference therein.

16. L. Wang, Y. Wang, X. G. Sun, J. Q. He, Z. Y. Pan, and C. H. Wang, Vacuum, 86 1174-1185 (2012).

17. Z. H. Xu, Y. C. Yang, P. Huang, and X. D. Li, Acta Mater., 58 5972-5979 (2010).

18. J. Y. Kwon, J. H. Lee, Y. G. Jung, and U. Paik, Surf. Coat. Technol., 201 3483-3490 (2006).

19. A. L. Ortiz, J. Sánchez-González, L. M. González-Méndez, and F. L. Cumbrera, Ceram. Int., 33 705-709 (2007).

20. N. A. Fleck, A. C. F. Cocks, and S. Lampenscherf, J. Eur. Ceram. Soc., 34 2687-2694 (2014).

21. K. Ma, J. G. Zhu, H. M. Xie, and H. X. Wang, Surf. Coat. Technol., 235 589-595 (2013)

22. T. Nakamura, G. Qian, and C. C. Berndt, J. Am. Ceram. Soc., 83 [3] 578584 (2000).

23. Q. Jiang, D. S. Zhao, and M. Zhao, Acta Mater., 49 3143-3147 (2001).

24. Z. Zhang, M. Zhao, and Q. Jiang, Semicond. Sci. Technol., 16 L33-L35 (2001).

25. D. C. Jia, Ceramic Materials, 2nd edition, eds., Y. Zhou. Science Publisher, Beijing, 320, 2004. 